\title{
Age Differences in State Curiosity: Examining the Role of Personal Relevance
}

\author{
Li Chu ${ }^{a, b}$ Helene H. Fung ${ }^{a}$ \\ ${ }^{a}$ Department of Psychology, The Chinese University of Hong Kong, Shatin, Hong Kong SAR; ${ }^{b}$ Department of \\ Psychology, Stanford University, Stanford, CA, USA
}

\section{Keywords}

Technology · Motivation · Self-relevance · Successful aging • Interests

\begin{abstract}
Objectives: Curiosity, or the desire for novel information and/or experience, is associated with improved well-being and more informed decisions, which has implications on older adults' (OAs') adoption of novel technologies. There have been suggestions that curiosity tends to decline with age. However, it was rarely studied under specific contexts, and there were relatively limited attempts to enhance OAs' curiosity. Under the theoretical framework of selective engagement theory, we examined age differences of curiosity in the context of learning a novel technology and investigated the moderating role of personal relevance. Method: This study utilized a pretest-posttest experimental design with a total of 50 younger adults (YAs) and 50 OAs from Hong Kong to measure their trait curiosity, perceived personal relevance, and state curiosity toward robots after interacting with a robot. Results: OAs showed significantly lower trait curiosity than YAs, but OAs showed a higher level of state curiosity toward a robot than YAs when they perceived an increase in personal relevance after interacting with the robot. Conclusion: Findings replicated previous findings that trait curios-
\end{abstract}

ity declined with age, but they also illustrated the distinctions between trait and state curiosity in the context of aging and highlighted the potential role of personal relevance in enhancing curiosity of OAs.

(c) 2021 The Author(s)

Published by S. Karger AG, Basel

\section{Introduction}

Intellectual wellness and proactive engagement are considered to be important dimensions of wellness for older adults (OAs) [1]. In other words, being open and curious toward novelties appears to be beneficial in later adulthood. Yet, this view raises a key question: Are OAs curious? This study aimed to understand the association between age and curiosity (defined as the desire to gain new information or experience). Specifically, this study focuses on curiosity toward novel technology, such as a robot, as this topic has profound implications for gerontechnological development and adoption. The study reported in this article aimed to test the role of a potential moderator suggested by the selective engagement theory. The moderator is personal relevance (i.e., whether one perceives a sense of connectedness between self and the target).

(C) 2021 The Author(s)

Published by S. Karger AG, Basel

This is an Open Access article licensed under the Creative Commons Attribution-NonCommercial-4.0 International License (CC BY-NC) (http://www.karger.com/Services/OpenAccessLicense), applicable to the online version of the article only. Usage and distribution for commercial purposes requires written permission.
Correspondence to:

Helene H. Fung, hhlfung @ psy.cuhk.edu.hk 


\section{Curiosity's Role across the Life Span}

Curiosity has long been considered a unique combination of emotion and motivation that drives informationseeking and exploratory behaviors [2]. According to Grossnickle [2], there are 2 types of curiosity: trait curiosity, which is an overall tendency to experience curiosity, and state curiosity, which is the variability of experiencing curiosity. These 2 types of curiosity have implications on people's well-being and decision-making.

First, trait curiosity seems to contribute to an individual's psychological well-being, social engagement, and physical health. In terms of psychological well-being, trait curiosity, especially the tendency to explore around, is positively associated with improved overall well-being [3]. Trait curiosity is also related to better social engagement. For instance, Kashdan and colleagues [4] found that people with more curious personality displayed more adaptive social behaviors, including more positive emotional expressiveness and less defensive attitudes. Interestingly, a link between trait curiosity and physical health was suggested by a longitudinal study where people with more curious disposition at baseline showed lower risk of mortality 5 years later [5]. These findings illustrate the importance of having curiosity for one's well-being.

Second, both trait and state curiosity are crucial to information processing, decision-making, and response initiation when in face of uncertainty. There has been growing evidence suggesting that the presence of state curiosity enhances information encoding and consolidation [6]. Curiosity can also affect decisions. A study on electricity and renewable energy decision revealed that the presence of curiosity influenced people's decision to install solar photovoltaics [7]. Moreover, curiosity appears to initiate more proactive responses. For example, individuals with more trait curiosity displayed a higher likelihood to self-initiate participation in a novel intervention [8] and to seek relevant information for better job performance [9]. Taken together, it seems important to have curiosity in order to facilitate decisions and motivation for change.

While most studies of curiosity focused on children, younger adults (YAs), and middle-aged adults, curiosity is also very important for and relevant to OAs. Later adulthood is a period of time that involves adaptation to many changes, such as personal illness and change of residence [10], where curiosity may play an essential role in enhancing decision-making and change initiation. Yet, as acknowledged in the review article by Sakaki et al. [11], curiosity in later adulthood is "one of the most underap- preciated topics on curiosity." Given curiosity's multidimensional nature, it would be useful to understand OAs' curiosity on a specific topic.

\section{A Topic That Deserves OAs' Curiosity: Novel Technology}

One topic that may greatly benefit OAs if they show more curiosity is "technologies." Gerontechnology, or technological innovations designed specifically for OAs, has gained increasing attention in industry, academia, and among the general public. According to a review article by Schulz et al. [12], novel technologies offer promising avenues to enhance OAs' lives in various domains, including physical and mental health, mobility, social connectedness, safety, and leisure. For instance, a qualitative study found that information and communication technologies, such as computers and mobile phones, not only optimized and improved older users' engagements in daily activities but also compensated for retirement and preserved important but distant social ties [13]. Novel technologies, such as robots, may also assist OAs' performance of daily activities ranging from housekeeping to social communication, and further improve OAs' autonomy and independence [14]. Such support appears to be particularly important in situations where face-to-face interactions are limited (e.g., living alone, living in rural areas, and during the COVID-19 pandemic).

Having interests in and usage of novel technologies may be beneficial in later adulthood. Yet, as the advancement of technology becomes more rapid, more technologies may be considered new and unfamiliar to OAs. Such unfamiliarity for OAs might be a concern because some studies found that OAs showed little interest and motivation in learning novel technologies [15]. Indeed, a factor that may prompt potential users to have the urge to learn more about or try out a novelty is "curiosity." In fact, one study found that curiosity moderated this association between motives and intention [16]. Furthermore, OAs with higher levels of trait curiosity were more likely to perceive novel technologies positively, which predicted higher likelihood of adoption and more frequent usage of these technologies [17]. In some sense, curiosity may be seen as the first step in novelty acceptance.

\section{Curiosity and Its Relationship with Age}

While curiosity may be important for novel technological acceptance in later adulthood, OAs seem to be less curious than YAs. Among the studies that examined aging and curiosity, there was no clear conclusion. On the one hand, a number of studies found that age was nega- 
tively associated with intellectual curiosity. Consistent with findings on an age-related decline of openness to experience, one study found a small but significant mean level decline of intellectual curiosity over a period of 5 years [18]. On the other hand, some studies did not find such relationship. For instance, Daffner et al. [19] used eye-tracking techniques and found that both middleaged and OAs showed similar levels of perceptual curiosity and exploratory visual movements. Similarly, Camp et al. [20] observed no significant age difference in state or trait curiosity during a word frequency-guessing task.

In a study by Dellenbach and Zimprich [21], they found that curiosity was associated with crystalized intelligence after controlling for age, sex, and educational level, suggesting that cognition is an important factor to consider in the context of curiosity. An experience sampling study also showed that engaging in curiosity-related activities (e.g., thinking for pleasure) could be cognitively demanding [22], which might contribute to people's disengagement from it. According to Hess' [23] selective engagement theory, age is associated with a decline in cognitive capacity, which contributes to disengagement from cognitively demanding tasks, including everyday activities such as volunteering, reading, or using a computer [24]. An earlier eye-tracking study illustrated that individuals with cognitive declines exhibited less perceptual curiosity and exploratory visual behaviors than people without cognitive declines [25]. In other words, with age and age-related cognitive changes, people are likely to disengage from cognitively demanding activities, such as being curious and engaged.

\section{Personal Relevance as a Moderator}

One of the key moderators on the association of age and engagement identified by this line of research was personal relevance. Hess and colleagues [26] found that OAs tended to allocate more cognitive resources in personally relevant situations and were less willing to engage cognitively in irrelevant situations. In a later study, Hess et al. [27] found that OAs were more likely to engage in systematic information search when the context was more personally relevant than when it was less personally relevant, but this pattern was not observed in YAs. They explained this observation in terms of OAs' adaptive preservation for their relatively limited cognitive resources.

In a review, Priniski et al. [28] defined personal relevance as a connection between a person and a stimulus that was personally meaningful to the person. They then highlighted 3 types of personal relevance along a continuum of increasing personal meaningfulness: personal as-

Age Differences in State Curiosity sociation, personal usefulness, and identification. Personal association captures a sense of connection between a stimulus and oneself and/or one's life; personal usefulness is the perception that a stimulus may help one to achieve personal goals; and identification is the incorporation of a stimulus into one's identity. We focused on the first 2 types of personal relevance in this article because identification would be more challenging to manipulate within a short period of time [28].

A study by Chien et al. [29] illustrated the importance of personal association, or the perception of connectedness between a target and oneself (e.g., whether a robot looks like oneself), in the context of curiosity and engagement with novel technologies. In this study, participants were instructed to interact with a robot; Zenbo developed by ASUS, with a given set of commands, and self-reported on the relevance after the interaction. Chien et al. [29] found that OAs had a lower level of state curiosity toward robots than YAs. They also found a significant interaction of personal association and age on state curiosity toward robots. Specifically, OAs showed a higher level of state curiosity when they perceived higher personal association, but this trend was not found among YAs.

Although the study by Chien and colleagues [29] provided support for the potential moderating role of personal relevance, all of the variables were measured after the direct interaction with a robot. This problem was addressed by Złotowski et al. [30]. They found that brief interactions with a robot that promoted perceived closeness seemed to have positive impacts on individuals' perception and attitudes of robots. These findings highlight the possibility to enhance personal association through direct interaction. The reported study in this article went one step further by directly examining the moderating role of personal relevance in the association between age and curiosity in the context of robot-related new technology.

\section{Current Study}

The overarching goal of this study is to examine if the framework of the selective engagement theory is applicable to curiosity. There were 2 specific aims: (1) to replicate the negative associations between age and state and trait curiosities as suggested in previous studies [15, 18 ] and (2) to investigate the moderating role of personal relevance on age and state curiosity in the context of novel technology. In this study, we predicted that the negative association between age and state curiosity would be diminished when personal relevance was higher. For both aims, we were particularly interested in state curiosity not only because it was more likely to be ma- 
Table 1. Means and standard deviations of key variables by age-groups

\begin{tabular}{|c|c|c|c|c|}
\hline Variable & YAs $(n=50)$ & OAs $(n=50)$ & $t$ & $p$ value \\
\hline Age, years & $22.76 \pm 3.82$ & $66.10 \pm 4.37$ & -52.82 & $<0.001$ \\
\hline State curiosity toward robot (pretest) & $4.01 \pm 1.09$ & $5.20 \pm 1.13$ & -5.31 & $<0.001$ \\
\hline State curiosity toward robot (posttest) & $4.27 \pm 1.18$ & $5.00 \pm 1.02$ & -3.33 & 0.001 \\
\hline Trait curiosity & $4.66 \pm 0.62$ & $4.08 \pm 0.63$ & 4.68 & $<0.001$ \\
\hline \multicolumn{5}{|l|}{$\Delta$ personal relevance, $n(\%)$} \\
\hline Increased & $35(70)$ & $25(50)$ & & \\
\hline No change & $10(20)$ & $10(20)$ & & \\
\hline Decreased & $5(10)$ & $15(30)$ & & \\
\hline \multicolumn{5}{|l|}{ Sex, $n(\%)$} \\
\hline Male & $19(38)$ & $20(40)$ & & \\
\hline Female & $31(62)$ & $30(60)$ & & \\
\hline \multicolumn{5}{|l|}{ Education, $n(\%)$} \\
\hline Below elementary school & 0 & 0 & & \\
\hline Elementary school & 0 & $4(8)$ & & \\
\hline High school & $1(2)$ & $32(64)$ & & \\
\hline Bachelor & $36(78)$ & $12(24)$ & & \\
\hline Masters & $9(18)$ & $2(4)$ & & \\
\hline Doctorate & $4(8)$ & 0 & & \\
\hline \multicolumn{5}{|l|}{ Monthly family income, ${ }^{*} n(\%)$} \\
\hline Less than USD 3,000 & $2(4)$ & $11(22)$ & & \\
\hline USD 3,001-8,500 & $1(2)$ & $4(8)$ & & \\
\hline USD $8,501-14,000$ & $3(6)$ & $4(8)$ & & \\
\hline USD $14,001-20,000$ & $3(6)$ & $8(16)$ & & \\
\hline USD $20,001-29,999$ & $16(32)$ & $8(16)$ & & \\
\hline USD $30,000-59,999$ & $16(32)$ & $9(18)$ & & \\
\hline USD 60,000-99,999 & $8(16)$ & $4(8)$ & & \\
\hline USD 100,000 or more & $1(2)$ & $2(4)$ & & \\
\hline
\end{tabular}

YAs, younger adults; OAs, older adults; HDK, Hong Kong dollars. Data are presented as $n$ (\%) or mean \pm

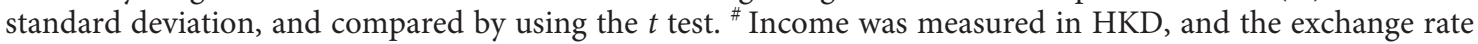
during the time of data collection was USD 1, to $\sim 7.82 \mathrm{HKD}$.

nipulated but also because arousing state curiosity seemed to influence the development of more stable and enduring trait curiosity toward the targeted topic [31]. We predicted that participants' personal relevance would be higher after interacting with the robot, which moderated the effect of age-group on state curiosity toward robots.

\section{Method}

To address the aims of this study, we conducted an experimental study that allowed participants to directly and freely interact with a robot. We measured participants' perceived personal relevance and state curiosity before and after the manipulation and human-robot interaction to see if changes of personal relevance across the interaction contributed to posttest state curiosity. This study paradigm was suggested by Kashdan and Fincham [32] as an effective strategy in promoting curiosity and creating personal relevance.

\section{Participants}

Before data collection, we conducted a power analysis for repeated ANOVA using G*Power [33] to determine the optimal sample size. We set the effect size to be 0.15 with 0.8 power and 0.05 alpha-error probability for a model that included 2 groups with 2 measurements. The analysis suggested that we needed at least a total sample of 90 people.

This study included a total of 50 YAs (female $=62.0 \%, M_{\text {age }}=$ 22.8 , age range $=19-34$ ) and $50 \mathrm{OAs}$ (female $=60.0 \%, M_{\mathrm{age}}=66.1$, age range $=60-78)$. We recruited younger participants from the participant pool of Department of Psychology, the Chinese University of Hong Kong. Older participants were recruited from the local communities in Hong Kong through (1) an existing participant list in the laboratory, (2) online advertisements via university mass mails, and (3) word of mouth. Due to the limitation of the stimulus (i.e., the robot we utilized in this study can only detect English commands) and the nature of the study, we screened for participants who could repeat simple English phrases and read Chinese characters. For other sample characteristics, see Table 1. As reimbursement, YAs received $1 \mathrm{~h}$ of participant pool credits and OAs received USD 200 HKD for their participation. 


\section{Materials}

In order to examine the impact of in-person interactions on personal relevance, we utilized a home robot, Vector, designed by Anki. This robot was designed to be a home companion with the capacity to detect voices, touches, faces, and depth. It is relatively small in size $(3.9 \times 2.4 \times 2.7$ inches $)$, but it has various functions (e.g., depth and edge perception, touch sensors, speech recognition, and distinct emotion expressions) that are relatively advanced for robots during the time of data collection. This robot was chosen for the purpose of the study because it was relatively affordable, displayed a number of emotions, offered functions in both social and practical domains, and was unknown to both YAs and OAs in Hong Kong.

This robot has a number of functions. In order to select the functions, we had 2 coders who independently categorized Vector's functions into social, problem-solving, or entertainment. Considering that YAs and OAs may have different preferences due to age-related goals and priorities [34], we selected 12 functions where the coders showed agreement in its categorization, which included 6 problem-solving functions (i.e., "What's the weather today?," "What time is it?," "Set a timer of $5 \mathrm{~s}$," "Take a picture of me," "I have a question, what is $2+2$ ?," and "Go to your charger") and 6 social functions (i.e., "Come here," "Give me a fist bump," "Bad robot," patting the robot's back, watching the robot flips itself, and "Be quiet"). These selected functions also allowed the robot to express different emotions, such as sadness, happiness, and anger.

\section{Measures}

All of the scales were translated and back-translated from English into Chinese. For the mean and standard deviation of each variable for this sample, see Table 1.

\section{State Curiosity toward Robot}

To measure participants' state curiosity toward the robot, we adapted a 3-item curiosity scale developed by Park et al. [35] into questions that targeted a specific robot. We also added 3 additional items using similar wordings as the original scale in order to capture more variations in participants' information-seeking tendencies. Together, our measure of state curiosity toward the robot included the following 6 questions: (1) "How curious do you feel about this robot?," (2) "How much do you want to know about this robot?," (3) "How likely would you be to spend time watching a short introduction clip of this robot?," (4) "If the user guide is easily accessible, how likely would you be to spend time reading these materials?," (5) "How likely would you be to spend time searching for this robot's information online on your own?," and (6) "If there is an advance course on how to use this robot, how likely would you be to spend time participating in this course?." These items were measured on a 7-point Likert scale ranging from 1 ("not at all") to 7 ("very much" or "very likely"). This measure's internal consistency was above the conventional standard (i.e., Cronbach's $a>0.70)$ for both the pretest (Cronbach's $\alpha=0.90$ ) and the posttest (Cronbach's $\alpha=0.87$ ).

\section{Personal Relevance}

An adapted version of an existing similarity scale [36] was utilized to capture personal relevance. These items were measured on a 5-point Likert scale ranging from 1 ("not similar at all") to 5 ("very similar"). For the purpose of this study, 3 items were selected to mea- sure personal association as they better captured the "humanlikeness" or personal association of the robot, which included the following: (1) "the degree to which you feel that this robot is similar to you in general," (2) "the degree to which you feel that this robot is similar to you in terms of appearance," and (3) "the degree to which you feel that this robot is similar to you in terms of personality." This measure had satisfactory internal consistency for both the pretest (Cronbach's $\alpha=0.74$ ) and the posttest (Cronbach's $\alpha=0.80$ ). Another 3 items focused more on the functionality of the robot and were used as manipulation check of the personal usefulness (another subtype of personal relevance) manipulation. Items included "I think the robot is useful to me," "It would be convenient for me to have the robot," and "I think the robot can help me with many things."

\section{Trait Curiosity}

Trait curiosity was measured using a 5-dimensional scale developed by Kashdan et al. [37] with a total of 25 items. It was on a 7 -point Likert scale ranging from 1 ("does not describe me at all") to 7 ("completely describes me") and the internal consistency was satisfactory (Cronbach's $\alpha=0.86$ ).

\section{Covariates}

A number of demographic variables, including age, sex, educational level, and income level, were included as covariates. We also examined participants' cognitive abilities using the 60 -s verbal fluency task and 90-s digit symbol task [38].

\section{Procedure}

After arriving at the laboratory and giving informed written consent, participants were asked to fill out the pretest measures in the following order: personal relevance, state curiosity toward robot, and trait curiosity. Questionnaires were presented on a tablet via Qualtrics. Since all of our participants have never seen or heard about the robot, we displayed an image of Vector at the beginning of the survey, so participants could refer to the robot when answering questions. We also presented a manipulation of personal usefulness at the end of the pretest questionnaire. Participants were randomly assigned to experimental, control, and waitlist control groups. In the experimental condition, participants read an article that described the prevalence of loneliness, linking its consequences to participants' age-group, and introduced Vector as a potential solution (i.e., personally relevant for the participant). In the control condition, participants read the same article, but the consequences were linked to the other age-group (i.e., not personally relevant for the participant). For the waitlist control group, participants read the same article, but the article did not mention the robot (i.e., no manipulation). After reading the manipulation article, participants filled out the personal relevance measures once again. Then, there was a learning phase and an interaction phase. During the learning phase, an experimenter demonstrated how to use the robot and invited participants to try out each of the 12 functions. During the interaction phase, participants were invited to play with Vector until they felt that it was enough, and we limited this phase to be no more than $30 \mathrm{~min}$ to ensure that each participant had roughly the same experience. Finally, participants were asked to fill out the posttest questionnaire, which included measures on personal relevance and state curiosity toward the robot, and completed the 2 cognitive tasks. In total, we measured personal relevance 3 times in order to capture changes in personal relevance right after the personal usefulness manipulation (com- 
Table 2. Fixed-effects ANOVA results using posttest curiosity as criterion, before controlling for condition

\begin{tabular}{lrrrrrrr}
\hline Antecedent & $\begin{array}{c}\text { Sum of } \\
\text { squares }\end{array}$ & df & $\begin{array}{l}\text { Mean } \\
\text { square }\end{array}$ & $F$ & $p$ value & $\begin{array}{c}\text { Partial } \eta^{2} \\
\begin{array}{l}\text { Partial } \eta^{2} \\
90 \% \text { CI [LL, UL] }\end{array}\end{array}$ \\
\hline Intercept) & 104.27 & 1 & 104.27 & 100.38 & 0.000 & \\
$\Delta$ personal relevance & 12.54 & 2 & 6.27 & 6.04 & 0.003 & 0.11 & {$[0.02,0.21]$} \\
Age-group & 0.05 & 1 & 0.05 & 0.04 & 0.833 & 0.00 & {$[0.00,0.02]$} \\
Age-group $\times \Delta$ personal relevance & 6.15 & 2 & 3.08 & 2.96 & 0.057 & 0.06 & {$[0.00,0.14]$} \\
Error & 96.61 & 93 & 1.04 & & & & \\
\hline
\end{tabular}

LL, lower limit of the partial $\eta^{2}$ confidence interval; UL, upper limit of the partial $\eta^{2}$ confidence interval.

Table 3. Fixed-effects ANOVA results using posttest curiosity as criterion, after controlling for condition

\begin{tabular}{lcrrrrrr}
\hline Antecedent & $\begin{array}{l}\text { Sum of } \\
\text { squares }\end{array}$ & df & $\begin{array}{l}\text { Mean } \\
\text { square }\end{array}$ & $F$ & $p$ value & $\begin{array}{c}\text { Partial } \eta^{2} \\
\text { Partial } \eta^{2} \\
90 \% \text { CI [LL, UL] }\end{array}$ \\
\hline Intercept) & 85.62 & 1 & 85.62 & 84.42 & 0.000 & \\
Condition & 3.29 & 1 & 3.29 & 3.25 & 0.075 & 0.03 & {$[0.00,0.11]$} \\
$\Delta$ personal relevance & 13.75 & 2 & 6.88 & 6.78 & 0.002 & 0.13 & {$[0.03,0.23]$} \\
Age-group & 0.21 & 1 & 0.21 & 0.20 & 0.654 & 0.00 & {$[0.00,0.04]$} \\
Age-group $\times \Delta$ personal relevance & 6.65 & 2 & 3.33 & 3.28 & 0.042 & 0.07 & {$[0.00,0.15]$} \\
Error & 93.32 & 92 & 1.01 & & & & \\
\hline
\end{tabular}

LL, lower limit of the partial $\eta^{2}$ confidence interval; UL, upper limit of the partial $\eta^{2}$ confidence interval.

paring the first and the second time) and changes in personal relevance after the interaction with robot (comparing the first and the third time). At the end of the session, each participant was reimbursed and debriefed of the purpose of the study.

\section{Statistical Analyses}

Manipulation check was performed with independent $t$ tests where conditions were entered as the independent variables and different types of personal relevance were the dependent variables. Next, to examine age-group differences in state and trait curiosity, we conducted independent sample $t$ tests, an ANOVA, and an analysis of covariance (ANCOVA) using R package "stats." In the $t$ test analyses, age-group was inputted as the independent variable, and trait and state curiosity were included as the dependent variables in separate analyses. In the ANOVA and ANCOVA, we used age-group (the independent variable), personal relevance (the moderator), and the interaction term (age group $\times$ personal relevance) to predict posttest state curiosity (the dependent variable). Typical covariates (i.e., sex, education, and income) were included in the $t$ test and ANCOVA.

\section{Results}

For the manipulation check, we performed independent $t$ tests to see if people in the experimental group perceived higher levels of personal usefulness right after reading the manipulation than before manipulation than people in the control or waitlist control group. The results were not significant, suggesting that the personal usefulness manipulation was not successful. However, we tested the impact of interaction with robot on personal association, and the paired $t$ test result was significant $(t$ [99] $=$ $3.70, p<0.001)$, indicating that compared to before the interaction $\left(M_{\text {pre }}=1.87\right)$, participants perceived higher levels of personal association after interacting with Vector $\left(M_{\text {post }}=2.24\right)$. From this point onward, we referred to personal association as personal relevance.

Next, we examined age differences in state and trait curiosity. For state curiosity, OAs $\left(M_{\text {pre }}=5.20 ; M_{\text {post }}=\right.$ 5.00) showed a significantly higher level of state curiosity toward the robot than YAs $\left(M_{\text {pre }}=4.01 ; M_{\text {post }}=4.27\right)$ for both the pretest $(t[97]=-5.31, p<0.001)$ and the posttest $(t[97]=-3.33, p=0.001)$. In terms of trait curiosity, OAs $(M=4.08)$ showed a lower level of trait curiosity than YAs $(M=4.66)$, and this difference was significant $(t[98]=$ 4.68, $p<0.001)$. The results remained unchanged even after controlling for sex, income, and educational level.

We then investigated the moderating role of personal relevance changes between pre- and posttests on the association between age and posttest state curiosity toward 
technology. We categorized personal relevance changes into 3 groups: increased, maintained (i.e., no change), and reduced. We found a marginally significant interaction between age-group and personal relevance changes on posttest state curiosity $(F[2,93]=2.96, p=0.057$, $\eta p^{2}=0.06$ ). After controlling for the manipulation conditions, the ANCOVA revealed a significant interaction $\left(F[2,92]=3.28, p=0.04, \eta p^{2}=0.07\right)$. For details of the 2 analyses, see Tables 2 and 3 , respectively. Simple main effects analysis revealed that when perceiving increased or maintained levels of personal relevance, $\mathrm{OAs}\left(M_{\text {increased }}=\right.$ 5.39; $\left.M_{\text {maintained }}=4.85\right)$ showed higher levels of curiosity than YAs $\left(M_{\text {increased }}=4.51 ; M_{\text {maintained }}=3.27\right)$, but no significant age difference was observed when perceived personal relevance was reduced over the interaction $\left(M_{\mathrm{YA}}=\right.$ $4.51 ; M_{\mathrm{OA}}=4.46$ ). Comparing within each age-group, for YAs, state curiosity levels were not significantly different when perceived personal relevance increased $(M=4.57)$ or reduced $(M=4.51, p>0.05)$. However, there was a marginally significantly higher level of state curiosity toward the robot when perceived personal relevance increased $(M=5.39)$ than reduced $(M=4.46)$ for OAs $(p=$ $0.06)$. For illustration, see Figure 1 . The results remained unchanged even after statistically controlling for sex, income, education, cognitive abilities, and pretest state loneliness (a single item asking how lonely the individual felt at the moment).

This study first replicated previous findings on aging and trait curiosity, that is, OAs reported a lower level of trait curiosity than YAs. However, different from prediction, OAs showed a higher level of state curiosity toward a robot than YAs. This study also provided support for the moderating role of personal relevance on OAs' curiosity toward a robot. Specifically, OAs showed a significantly higher level of state curiosity when their perceived personal relevance increased rather than reduced after the human-robot interaction, but this pattern was not found among YAs.

\section{Discussion}

The study reported herein further evidence for the importance of personal relevance in the context of promoting curiosity and engagement among OAs. First, while trait curiosity showed a negative association with age in the current and previous studies $[15,18]$, the relationship between age and state curiosity appeared to be reversed, possibly due to its momentary nature. Specifically, OAs showed more state curiosity toward a robot than YAs.

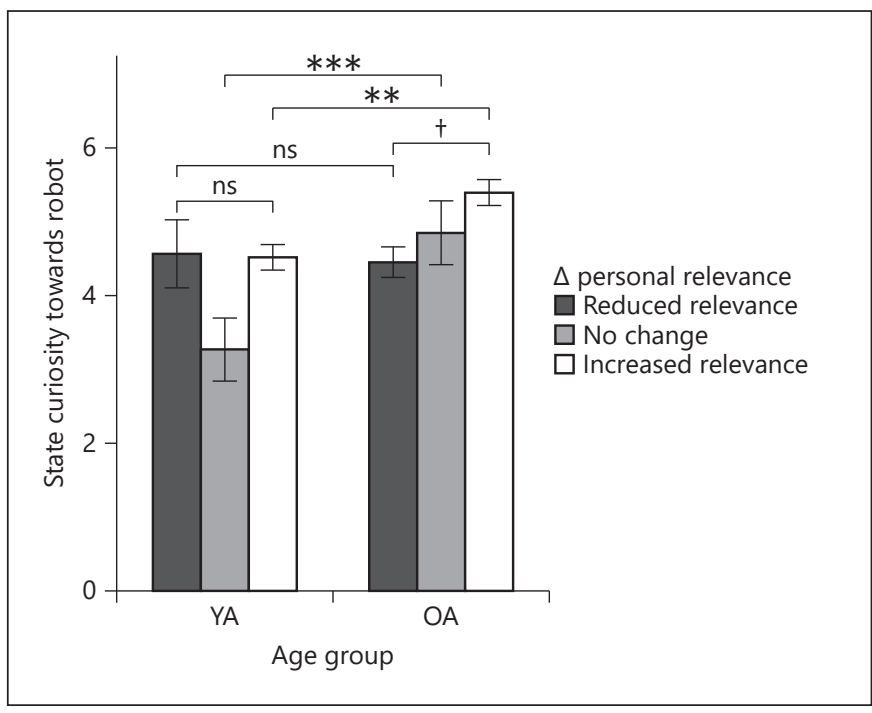

Fig. 1. Bar graph of the age $\times$ personal relevance interaction. YAs, younger adults; OAs, older adults.

While our result on state curiosity differs from our expectation, it illustrates a specific context where OAs report greater curiosity than YAs. This finding opens up the opportunity for future aging research to target momentary states of curiosity, which might be more malleable and could eventually shape trait curiosity [31]. Second, this study offered support for the moderating role of personal relevance on OAs' state curiosity toward novelties, which was consistent with the selective engagement theory and other related studies (e.g., [27]). Specifically, our study strengthened previous findings by directly manipulating personal relevance through human-robot interactions and testing its effect on curiosity.

Findings from the current study add to the aging literature regarding the impacts of direct interactions on personal relevance (in terms of personal association) in the context of novel technologies. They show that interactions with novelties could increase personal relevance, making OAs as curious about the novelties as YAs. Previous works showed that people were more likely to be curious if they perceived stimuli to be new, unexpected, and complex, rather than something familiar [39]. However, the current study presents contrasting findings that OAs may be more curious when they perceive stimuli to be somewhat familiar or connected to self, suggesting a new direction to increase the curiosity of OAs.

The present study bears some limitations. We acknowledge that without random sampling, our study's results were subjected to selection biases. For instance, par- 
ticipants who showed interests and participated in a technology-related study might be more curious overall or specifically about technologies than the general public. Future studies should attempt to replicate our findings in different contexts with more representative samples. Moreover, the manipulation check showed that although interacting with a robot increased perceived personal association (one personal relevance subtype) with the robot, reading an article that described the personal usefulness of a robot to reduce loneliness in one's age-group did not increase perceived personal usefulness (another personal relevance subtype) of the robot. These results may suggest that direct interaction is a more effective means to prime personal relevance than passive viewing of an article. They may also suggest that personal association is easier to manipulate than personal usefulness. Future studies should seek to enhance personal relevance through more direct means. For example, one study showed that explicit prompts that guide participants to pause and think for a few minutes after an activity helped them enjoy their own thoughts and perceive the activity to be personally meaningful [40]. Priming studies also demonstrated that priming different values (e.g., security vs. self-direction) or different goals (e.g., learning) changed individuals' curiosity behaviors [41]. Future studies may consider using similar paradigms to enhance perceived personal relevance and ultimately enhance OAs' curiosity, without direct interaction. We also argue that personal relevance may serve as an important index for assessing the success of human-robot interactions in future studies. Finally, we acknowledge that we did not measure participants' prior experience with technologies, so we cannot exclude its potential influence on our results.

Despite these limitations, our study has important implications for future technology designs, aging interventions, and aging research on curiosity. First, future gerontechnologies may consider incorporating a sense of personal relevance in designs. For instance, it may be helpful to make the appearance of a novel device resemble something that OAs may be more familiar with (e.g., an older technology or more humanlike). Second, even though trait curiosity may decline with age, state curiosity is more malleable. Knowing that curiosity has been associated with various positive outcomes as mentioned in the Introduction section, care providers and local organizations should continue efforts in enhancing OAs' state curiosity toward novelties and diversities. Third, such state curiosity appears to be a promising first step in OAs' acceptance of novelty. Future studies should examine the long-term impacts of curiosity on technological acceptance and novelty learning. Undeniably, old age is accompanied by various changes that may be seen as declines and interpreted as negative, but it can also be an opportunity for growth if we can enhance curiosity through increasing personal relevance.

\section{Statement of Ethics}

This study was approved by the Survey and Behavioural Research Ethics Committee of the Chinese University of Hong Kong (Ref No. 4052175). This study complied with the guidelines for human studies and was conducted ethically in accordance with the World Medical Association Declaration of Helsinki. All participants provided written informed consent before participating in this study.

\section{Conflict of Interest Statement}

The authors have no conflicts of interest to declare.

\section{Funding Sources}

This project was funded by a General Research Fund (RGC Ref No. 14612718) from the Research Grants Council (RGC), Hong Kong, and a direct grant for research from the Faculty of Social Sciences of the Chinese University of Hong Kong to Prof. Helene H. Fung.

\section{Author Contributions}

Both authors have fulfilled the ICMJE Criteria for Authorship and contributed to the design, data collection, data interpretation, writing, and approval of this study.

\section{References}

1 Lee JE, Kahana B, Kahana E. Successful aging from the viewpoint of older adults: development of a brief successful aging inventory (SAI). Gerontology. 2017;63:359-71.

2 Grossnickle EM. Disentangling curiosity: dimensionality, definitions, and distinctions from interest in educational contexts. Educ Psychol Rev. 2016;28:23-60.

3 Gallagher MW, Lopez SJ. Curiosity and wellbeing. J Posit Psychol. 2007;2(4):236-48.

4 Kashdan TB, Sherman RA, Yarbro J, Funder DC. How are curious people viewed and how do they behave in social situations? From the perspectives of self, friends, parents, and unacquainted observers. J Pers. 2013;81(2):142-54.

5 Swan GE, Carmelli D. Curiosity and mortality in aging adults: a 5-year follow-up of the Western Collaborative Group Study. Psychol Aging. 1996;11(3):449-53. 
6 Gruber MJ, Ranganath C. How curiosity enhances hippocampus-dependent memory: the prediction, appraisal, curiosity, and exploration (PACE) framework. Trends Cogn Sci. 2019;23(12):1014-25.

7 Chapman A, Itaoka K. Curiosity, economic and environmental reasoning: public perceptions of liberalization and renewable energy transition in Japan. Energy Res Soc Sci. 2018; 37:102-10.

8 Kaczmarek LD, Kashdan TB, Drazkowski D, Bujacz A, Goodman FR. Why do greater curiosity and fewer depressive symptoms predict gratitude intervention use? Utility beliefs, social norm, and self-control beliefs. Pers Individ Dif. 2014;66:165-70.

9 Harrison SH, Sluss DM, Ashforth BE. Curiosity adapted the cat: the role of trait curiosity in newcomer adaptation. J Appl Psychol. 2011;96(1):211-20.

10 Hardy SE, Concato J, Gill TM. Stressful life events among community-living older persons. J Gen Intern Med. 2002;17(11):832-8.

11 Sakaki M, Yagi A, Murayama K. Curiosity in old age: a possible key to achieving adaptive aging. Neurosci Biobehav Rev. 2018;88:10616.

12 Schulz R, Wahl HW, Matthews JT, De Vito Dabbs A, Beach SR, Czaja SJ. Advancing the aging and technology agenda in gerontology. Gerontologist. 2015;55(5):724-34.

13 Nimrod G. Aging well in the digital age: technology in processes of selective optimization with compensation. J Gerontol Ser B. 2019; gbz111:1-10.

14 Rogers WA, Mitzner TL. Envisioning the future for older adults: autonomy, health, wellbeing, and social connectedness with technology support. Futures. 2017;87:133-9.

15 Stamato C, Quaresma M, Mont'Alvão CR. Aging and new technologies: challenges and perspectives. In: Marcus A, editor. Design, user experience, and usability user experience design practice DUXU 2014 lecture notes in computer science. Berlin, Germany: Springer; 2014. p. 173-84

16 Koo DM, Choi YY. Knowledge search and people with high epistemic curiosity. Comput Human Behav. 2010;26(1):12-22.

17 Gilly MC, Celsi MW, Schau HJ. It don't come easy: overcoming obstacles to technology use within a resistant consumer group. J Consum Aff. 2012;46(1):62-89.
18 Mascherek A, Zimprich D. Stability and change in typical intellectual engagement in old age across 5 years. J Gerontol B Psychol Sci Soc Sci. 2012;67(3):309-16.

19 Daffner KR, Scinto LFM, Weintraub S, Mesulam M-M. The impact of aging on curiosity as measured by exploratory eye movements. Arch Neurol. 1994;51:368-76.

20 Camp CJ, Dietrich MS, Olson KR. Curiosity and uncertainty in young, middle aged, and older adults. Educ Gerontol. 1985;11(6):40112.

21 Dellenbach M, Zimprich D. Typical intellectual engagement and cognition in old age. Aging Neuropsychol Cogn. 2008;15(2):208-31.

22 Westgate EC, Wilson TD, Gilbert DT. With a little help for our thoughts: making it easier to think for pleasure. Emotion. 2017;17(5):82839.

23 Hess TM. Selective engagement of cognitive resources: motivational influences on older adults' cognitive functioning. Perspect Psychol Sci. 2014;9(4):388-407.

24 Queen TL, Hess TM. Linkages between resources, motivation, and engagement in everyday activities. Motiv Sci. 2018;4(1):26-38.

25 Daffner KR, Scinto LFM, Weintraub S, Guinessey JE, Mesulam MM. Diminished curiosity in patients with probable Alzheimer's disease as measured by exploratory eye movements. Neurology. 1992;42(2):320-8.

26 Hess T, Rosenberg DC, Waters SJ. Motivation and representational processes in adulthood: the effects of social accountability and information relevance. Psychol Aging. 2001;16(4): 629-42.

27 Hess TM, Queen TL, Ennis GE. Age and selfrelevance effects on information search during decision-making. J Gerontol Psychol Sci. 2013;68(5):703-11.

28 Priniski SJ, Hecht CA, Harackiewicz JM. Making learning personally meaningful: a new framework for relevance research. J Exp Educ. 2018;86(1):11-29.

29 Chien S-E, Chu L, Lee H, Yang C, Lin F, Yang $\mathrm{P}$, et al. Age difference in perceived ease of use, curiosity and implicit negative attitude toward robots. ACM Trans Human Robot Interact. 2019;8(2):1-19.
30 Złotowski JA, Sumioka H, Nishio S, Glas DF, Bartneck C, Ishiguro H. Persistence of the uncanny valley: the influence of repeated interactions and a robot's attitude on its perception. Front Psychol. 2015;6(883):1-13.

31 Rotgans JI, Schmidt HG. Interest development: arousing situational interest affects the growth trajectory of individual interest. Contemp Educ Psychol. 2017;49:175-84.

32 Kashdan TB, Fincham FD. Facilitating curiosity: a social and self-regulatory perspective for scientifically based interventions. In: Linley A, Joseph S, editors. Positive psychology in practice. Hoboken, NJ: John Wiley \& Sons, Inc.; 2004. p. 482-503.

33 Faul F, Erdfelder E, Lang AG, Buchner AG. $\mathrm{G}^{*}$ power 3: a flexible statistical power analysis program for the social, behavioral, and biomedical sciences. Behav Res Methods. 2007; 39(2):175-91.

34 Carstensen L. The influence of a sense of time on human development. Science. 2006; 312(5782):1913-6.

35 Park SH, Mahony DF, Kim Y, Kim YD. Curiosity generating advertisements and their impact on sport consumer behavior. Sport Manag Rev. 2015;18(3):359-69.

36 Lutz-Zois CJ, Bradley AC, Mihalik JL, Moorman-Eavers ER. Perceived similarity and relationship success among dating couples: an idiographic approach. J Soc Pers Relat. 2006; 23(6):865-80.

37 Kashdan TB, Stiksma MC, Disabato DD, McKnight PE, Bekier J, Kaji J, et al. The fivedimensional curiosity scale: capturing the bandwidth of curiosity and identifying four unique subgroups of curious people. J Res Pers. 2018;73(January 2018):130-49.

38 Lezak M. Neuropsychologial assessment. 3rd ed. New York, NY: Oxford University Press; 1995.

39 Silvia P. Appraisal components and emotion traits: examining the appraisal basis of trait curiosity. Cogn Emot. 2008;22(1):94-113.

40 Alahmadi S, Buttrick NR, Gilbert DT, Hardin AM, Westgate EC, Wilson TD. You can do it if you really try: the effects of motivation on thinking for pleasure. Motiv Emot. 2017; 41(5):545-61.

41 Chen X, Latham GP. The effect of priming learning vs. performance goals on a complex task. Organ Behav Hum Decis Process. 2014; 125(2):88-97. 